\title{
Effect of Telenursing on Outcomes of Provided Care by Caregivers of Patients With Head Trauma After Discharge
}

\author{
Akram Shahrokhi, MSc $\square$ Jalil Azimian, PhD $\square$ Atousa Amouzegar, MSc $\square$ Sonia Oveisi, PhD
}

\begin{abstract}
Telenursing is a suitable tool for increasing health-related awareness of the caregivers for a better home care. But its efficacy may be affected by several factors. Considering the important complications of head trauma injury and high rate of readmission, we aimed to assess the effect of telenursing on care provided by the family members of patients with head trauma.

This randomized controlled trial investigated 72 patients with head trauma, who were randomly allocated to intervention and control groups (36 patients in each group). The caregivers in both groups were provided with 1-hr face-to-face training session on patients' home care and educational booklets. The patients in the intervention group were followed up every week through phone calls by the telenurse for 12 weeks, who recorded the patient's status, as well. Caregivers in the intervention group could call the telenurse any time they desired. The health status of the control group was followed once by a phone call after 12
\end{abstract}

ach year, about 1.7 million head traumas occur in the United States, leading to nearly 52,000 deaths and 275,000 hospitalizations (Hinkle \& Cheever, 2014), about 1.1 million of whom are treated and discharged from hospital (Brunner, Smeltzer, Bare, Hinkle, \& Cheever, 2010), whereas a considerable number $(80,000-90,000)$ will suffer from long-term disabilities (Hinkle \& Cheever, 2014). During 2012-2013, from 55,427 individuals who referred with head trauma to three main trauma centers in Tehran, mainly $(74 \%)$ consisting of men, the main causes of trauma included road accidents, falls, and violence ( Aligholi \& Safahani, 2015).

Author Affiliations: Department of Critical Care Nursing (Mr Shahrokhi), Nursing \& Midwifery Faculty ( $\mathrm{Dr}$ Azimian), Critical Care Nursing Department, Nursing \& Midwifery School (Ms Amouzegar), and School of Nursing and Midwifery (Dr Oveisi), Qazvin University of Medical Sciences, Qazvin, Iran.

The authors declare no conflicts of interest.

Correspondence: Atousa Amouzegar, MSc, Critical Care Nursing Department, Nursing \& Midwifery School, Qazvin University of Medical Sciences, Qazvin, Iran 3414667166 (atousa.amouzgar@gmail.com).

DOI: 10.1097/JTN.0000000000000338 weeks. Data on patients' readmission and pressure ulcer (based on Norton's scale) rate and time were compared between the groups and analyzed using SPSS software, version 19 . Thirty-three patients with a mean $\pm S D$ age of $31.12 \pm 10.83$ years were studied in the control group and 35 patients with a mean $\pm S D$ age of $34.11 \pm$

12.34 years in the intervention group ( $p=.098$ ). None of the patients in the intervention group were readmitted, whereas 2 patients in the control group were readmitted $\mathrm{s}(p=.139)$. Risk of pressure ulcer did not differ between the groups ( $p=.583$ ). Telenursing had no significant effect in readmission and decubitus prevention for patients with head trauma. Considering the chronic nature of the illness, a longer follow-up period is deemed necessary for an accurate conclusion.

\section{Key Words}

Caregivers, Head injury, Outcome assessment, Patient care, Telenursing

Head trauma can lead to several complications, ranging from mild concussions to coma and death (Hinkle \& Cheever, 2013). Traumatic brain injury (TBI) is the leading cause of mortality and morbidity worldwide and imposes a great burden on the health care system (Langlois, Rutland-Brown, \& Wald, 2006; Vafaee et al., 2013). The most common complications after head trauma and brain injury include coma, urinary and respiratory tract infections, septicemia, wound injury, meningitis, and brain abscess (Białkowska, Mariusz, \& Wojciech, 2012; Cheever, Hinkle, $\&$ Hinkle, 2014). Many of the head trauma survivors live with major disabilities, including physical, cognitive, mental, emotional, and behavioral defects (Haffejee, Ntsiea, \& Mudzi, 2013; Vafaee et al., 2013). These complications in patients with TBI increase the possibility of readmission to $7 \%, 12.7 \%$, and $17.6 \%$ after 1,3 , and 6 month(s), respectively (Boutin et al., 2013). Therefore, follow-up is a major part of health care services for these patients. A consistent and dynamic health care relationship with patients will increase the knowledge and function of patients and their families (Zakerimoghadam, Bassampour, Rjab, Faghihzadeh, \& Nesari, 2009). 